\title{
A METHOD FOR GENERATING NON-GAUSSIAN NOISE SERIES WITH SPECIFIED PROBABILITY DISTRIBUTION AND POWER SPECTRUM
}

\author{
Shen Minfen* Francis H. Y. Chan** Patch J. Beadle*** \\ *Science Research Center, Shantou University, Guangdong 515063, China \\ Email: mfshen@stu.edu.cn, Fax:+86-754-2510655 \\ **Department of Electronic Engineering, Hong Kong University, Hong Kong. \\ ***School of System Engineering, Portsmouth University, Portsmouth, U.K.
}

\begin{abstract}
It is necessary to generate a non-Gaussian noise sequence in the simulation of the communication system and signal processing. In the design of some practical system such as radar system, we must generate a stationary noise sequence or clutter series with a specified non-Gaussian probability density function and a desired power spectrum for the purpose of testing the performance of the system. This paper presents a novel method by which such a non-Gaussian noise sequence can be generated. The simulation results are also demonstrated to show the effectiveness of the proposed method.
\end{abstract}

\section{INTRODUCTION}

In the simulation and test of the communication systems in the applications of radar, communication as well as the signal processing it is necessary to provide a noise sequence or clutter series with required non-Gaussian probability density function, such as Weibull distribution or Rayleigh distribution, and the desired power spectrum in order to improve the performance of the systems and the behavior of the signal detection [1-2]. Generally, some methods have been introduced to transform a random sequence with a uniform distribution on the interval $[0,1]$ into a Gaussian random sequence with a specified autocorrelation or spectrum. In practical applications, however, it turns out to be very important to generate a stationary non-Gaussian random sequence with not only: a specified probability density function, but also a specified power spectrum for the purpose of radar system design and the behavior test. Therefore, we need to find the effective way to produce such a non-Gaussian random sequence in the practical situations. For this purpose, one procedure with linear and nonlinear systems in this paper is developed to generate a noise sequence or clutter series which has a specified non-Gaussian probability density function and the desired power spectral density. The simulated results for both probability distribution function and spectrum are also demonstrated to prove the effectiveness of the presented method. Finally, some significant results are discussed and summarized.

\section{METHOD}

The basic scheme for generating such a random sequence with specified distribution function and desired power spectral density is shown in the following block diagram:

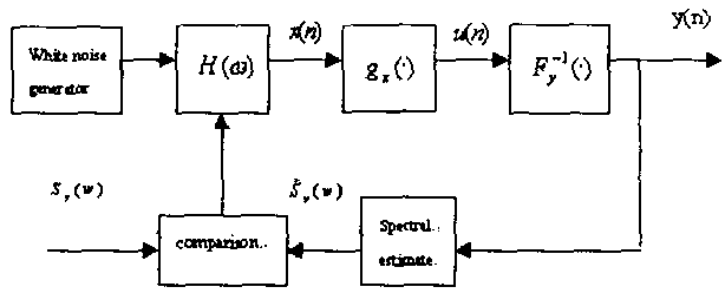

Fig. 1. The block diagram for transforming a white Gaussian noise into a desired sequence.

The output $y(n)$ represents the desired non-Gaussian noise sequence which has the probability distribution 
function $F_{y}(\cdot)$ and the spectral density function $S_{y}(f)$. There are three parts involved in the Fig. 1: (1) the linear system $H(z)$. (2) the nonlinear system $g_{x}(\cdot)$ and $F_{y}{ }^{-1}(\cdot) \cdot(3)$ the feedback part. First of all, as the input of the linear system, the output of the white noise generator $w(n)$ is a zero-mean unit-varance white Gaussian noise. $H(z)$ denotes the transfer function of the linear system which is designed to transform a white Gaussian noise sequence $w(n)$ into a Gaussian sequence with the desired spectral density function based on the fact that a linear transform of a Gaussian process produces another Gaussian process [3]. It is possible to assume that the variance of $x(n)$ can be scaled to unit-variance by normalizing the coefficients of linear digital filter [1]. Next, the nonlinear system $G($ ) includes the normalized Gaussian distribution function $g_{x}(\cdot)$ and the inverse of the distribution function $F_{y}{ }^{-1}(\cdot)$ of the desired random sequences $z(n)$. $g_{x}(\cdot)$, the first part of the nonlinear system, is used for transforming the Gaussian sequences $x(n)$ into a random sequence $u(n)$ which is normally distributed in the unit interval [7], and because $F_{y}{ }^{-1}(\cdot)$ is equal to the inverse of the probability distribution function of the desired sequences $y(n)$ so that the output sequences $y(n)$ will have the specified probability distribution function. Besides, the desired power spectrum of the random sequences $y(n)$ must be satisfied. The nonlinear system consists of the normalized Gaussian distribution function followed by a $F_{y}{ }^{-1}(\cdot)$ function and can be expressed as

$$
g(x)=F_{z}^{-1}\left[G_{y}(\cdot)\right]
$$

If the auto-correlation function or the spectrum of the desired sequences $y(n)$ has been specified, the auto-correlation function $R_{x}(m)$ can be decided through the transform of the nonlinear system. Once the $R_{x}(m)$ is obtained, all the coefficients of the linear system $H(z)$ can be definitely determined. Thus, the proposed system in Fig. 1 has been described such that when a Gaussian white noise sequence is used as an input into the system, the output sequences $y(n)$ will be a stationary non-Gaussian sequence with specified probability distribution function and power spectrum by the transform of both the linear and the nonlinear systems. Finally, to obtain a more accurate power spectrum in the output of the system, a feedback procedure is proposed to compare the spectral estimate of the $y(n)$ with the specified spectrum $S_{y}(\omega)$ and to adjust the parameters of the linear system via the error of the spectral estimate $\hat{S}_{y}(\omega)$.

\section{IMPLEMENTATION}

Based on the model in Fig. 1, it can be seen that the nonlinear function $G(\cdot)$ is described provided the probability distribution function $F_{y}(\cdot)$ has been definitely provided. It is further assumed that the nonlinear function can be expanded into the Hermite polynomials [1-2]

$$
G(X)=\sum_{K=0}^{\infty} b_{K} H_{K}(X)
$$

where $H_{K}(x)$ represents the $K$ th Hermite polynomial which is represented by

$$
H(X)=(-1)^{K} e^{x^{2}} \frac{d^{K}}{d X^{K}} e^{-X^{2}}
$$

and there exists the following relationship

$$
H_{K+1}=2 x H_{K}(X)-2 K H_{K-1}(X)
$$

The coefficients of Hermite polynomial $b_{K}$ are given by

$$
b_{K}=(K-1)^{-1} \int_{-\infty}^{\infty} g(X) H_{K}(X) f(X) d X
$$

where $f(X)$ is the normalized Gaussian density function. Since $x(n)$ can be normalized into a standard Gaussian sequence, the relationship of the auto-correlation between the input and output of the nonlinear system can be described as [1]

$$
R_{y}(m)=\sum_{K=1}^{\infty} C_{K}^{2} R_{x}^{K}(m)
$$


where the coefficients $C_{K}$ can be determined

$$
C_{K}=(K-1)^{-1 / 2} \int_{-\infty}^{\infty} g(X) H_{K}(X) f(X) d X
$$

Once the $C_{K}$ is computed and $R_{y}(m)$ is specified, the $R_{x}(m)$ can be found by solving the equation (6). In practical simulation, it is possible and applicable to choose the order of equation (6) to be finite because the $C_{K}$ has the property

$$
\sum_{k=1}^{\infty} C_{k}=1
$$

Furthermore, for majority of probability distribution functions, there exists the following approximate relation [2][6]

$$
\sum_{k=1}^{p} C_{K}=1
$$

Then the equation (6) can be expressed as

$$
R_{y}(m)=\sum_{k=1 y}^{p} C_{K}^{2} R_{x}^{K}(m)
$$

In order to determine the $R_{x}(m)$ from the given $R_{y}(m)$, a simple iterative procedure for calculating $R_{x}(m)$ from the given $R_{y}(m)$ derived from equation (8) is given such that

$$
R_{x}(m+1)=R_{y}(m)+\frac{u A(m)}{B(m)}
$$

where

$$
\begin{gathered}
A(m)=R_{y}(m)-\sum_{K=1}^{P} C_{X}^{2} R_{x}^{K}(m) \\
B(m)=\sum_{X=1}^{P} K C_{K}^{2} R_{x}^{K-i}(m)
\end{gathered}
$$

and $u$ is a convergent constant to be adjusted.

Once $R_{x}(m)$ has been decided, there are several methods for finding the coefficients of the linear system $H(z)$ [1,4-5]. In this paper, rather than use the conventional filtering techniques, we propose the AR model with order $\mathrm{N}$ as the linear system to provide the
Gaussian sequence with required auto-correlation $R_{x}(m)$. The AR model is equivalent to the maximum entropy method for spectral estimation $\hat{S}_{x}(\omega)$ and makes the calculation of the parameters of the $A R$ model more convenient and effective.

Since the input of the linear system is a white Gaussian noise sequence $w(n)$, the AR model can be written by

$$
y(n)=-\sum_{k=1}^{N} a_{K} y(n-K)+w(n)
$$

where $a_{K}$ is the model parameters to be decided. Based on the AR model, the auto-correlation function $R_{x}(m)$ can be described as

$$
R_{y}(m)= \begin{cases}-\sum_{K=1}^{N} a_{K} R_{y}(m-K), & m>0 \\ -\sum_{K=1}^{N} a_{K} R_{y}(m-K)+c, & m=0\end{cases}
$$

which is the well-known expression of Yule-Walker equation, and the model parameters can be estimated by solving the Yule-Walker equation after selecting the appropriate order $N$ of the AR model [6]. So far, both linear and nonlinear systems in Fig. 1 have been described and determined. Hence, if a white Gaussian noise sequence $w(n)$ is driven to the linear system followed by the nonlinear system including $g_{x}(\cdot)$ and $F^{-1}(\cdot)$, the output of the whole system will be such a noise sequence having a specified non-Gaussian probability distribution function $F_{y}(z)$ and a required auto-correlation function or spectrum $S_{y}(\omega)$, respectively.

\section{SIMULATION RESULTS}

A random sequence $z(n)$ with an exponential probability density function $p_{t}(z)$ and an exponential auto-correlation function $R_{z}(m)$ is generated by using the proposed method. The simulated results are shown in Fig. 2. The specified probability density function is shown with the solid line and the simulation result is indicated with the star symbols in Fig. 2 (a). In addition, in Fig. 2 (b), the required auto-correlation is shown 
with the solid line and the estimate result is demonstrated with the star symbols. The simulation shows that the estimate results of the non-Gaussian noise generated is consistent with the specified results in both probability, density function and auto-correlation functions.

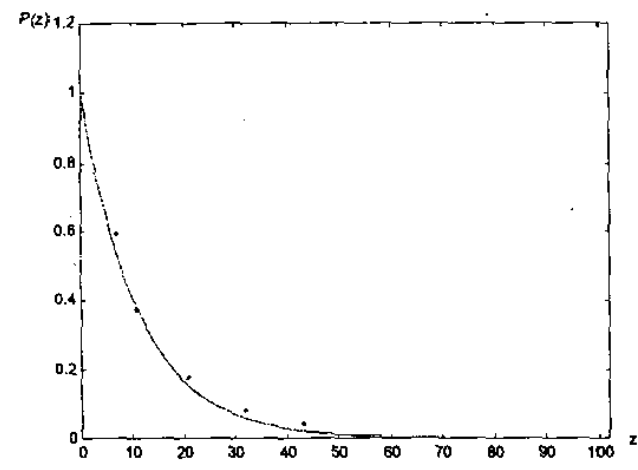

(a)

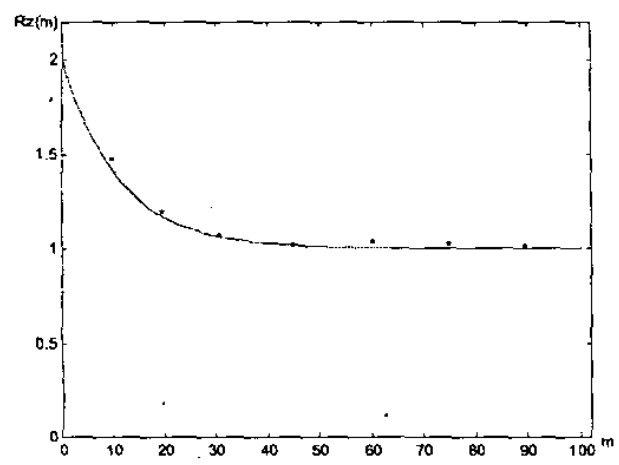

(b)

Fig.2 An example of a random sequence with a specified exponential probability density function (a) and an exponential auto-correlation function (b). The continuous curves denote the specified probability density function and the auto-correlation, respectively. The star symbols represent the simulation results based on the proposed approach.

Unlike the conventional filtering approach, the AR model enable us to provide a Gaussian noise with the required spectrum easily and accurately. Moreover, with the proposed feedback scheme, the spectral estimation of the non-Gaussian noise sequence was compared with the specified power spectral density to obtain a spectral estimation error which was employed as the output of the feedback system to adjust the parameters of the AR model so that the spectral estimation error can be controlled to a desired range.

\section{CONCLUSION}

This contribution is devoted to describing a novel approach for generating of a non-Gaussian noise sequence with both a specified probability distribution function and a required power spectrum. Our procedure consists of an AR model with a Gaussian white noise sequence input followed by a nonlinear system, which transforms the output sequences of the linear system into such a random sequence with not only a specified probability density function but also a desired auto-correlation function, respectively. In order to obtain a more accurate spectral estimation of the noise sequence generated, a feedback system was implemented to compare the spectral estimate with the specified spectrum of the non-Gaussian noise sequence such that the output of the comparison result was employed to adjust the parameters of the AR model. The method presented in this paper has shown the advantages of simplicity and applicability in many applications of communications and radar system analysis.

\section{REFERENCES}

[1] Shen J., Approach of a method for generating a random sequence, Technique of Fire-Controlled Radar, No.3, 1986.

[2] S. Shlien, real time generation of narrow-band audio noise, IEEE Signal Processing Letter, Vol.3(4), 1996.

[3] Whalen A. D., Detection of Signals in Noise, Academic Press, Inc, 1971.

[4] Haykin S., Nonlinear Method of Spectral Analysis, Springer-Verlay, 1983.

[5] Liu B. and Munson D.C., Generation of a random sequence having a jointly specified marginal distribution and auto-covariance, IEEE Trans. ASSP-30, No. 6, December, 1982

[6] Papoulis A., Probability, Random Variables, and Stochastic Processes, New York: McGraw-Hill, 1965. 